Article

\title{
Four Types of Fixed-Point Theorems for Multifunctions in Probabilistic Metric Spaces ${ }^{\dagger}$
}

\author{
Endre Pap (D)
}

Citation: Pap, E. Four Types of Fixed-Point Theorems for Multifunctions in Probabilistic Metric Spaces. Mathematics 2021, 9, 3212. https://doi.org/10.3390/math 9243212

Academic Editor: Vilém Novák

Received: 23 November 2021 Accepted: 9 December 2021 Published: 12 December 2021

Publisher's Note: MDPI stays neutral with regard to jurisdictional claims in published maps and institutional affiliations.

Copyright: (C) 2021 by the author. Licensee MDPI, Basel, Switzerland. This article is an open access article distributed under the terms and conditions of the Creative Commons Attribution (CC BY) license (https:/ / creativecommons.org/licenses/by/ $4.0 /)$.
Singidunum University, 11000 Belgrade, Serbia; epap@singidunum.ac.rs

+ This paper is dedicated to the memory of my good friend Olga Hadžić, a lady with a big heart and a mathematician with nice original ideas.

\begin{abstract}
An overview of fixed-point theorems (F.P.T.s) for multifunctions in probabilistic metric spaces is given. Extensions of the fixed-point theorems on probabilistic metric spaces of Nadler, Hadžić, Itoh, and Miheţ are presented. In the end, some hints about some further related investigations are given.
\end{abstract}

Keywords: triangular norm; probabilistic metric space; multivalued function; fixed-point theorem

MSC: 47H10, 46S50, 54H25

\section{Introduction}

Linear functional analyses were elaborated in the first half of the last century. However, it turns out that there are many problems that are nonlinear and that require a new theory. Fixed-point theory is very useful in mathematics and especially as the basic tool of nonlinear analysis [1-5].

The theory of multivalued functions (set-valued maps) is an important part of theoretical investigations [6,7] and practical applications [6,8,9]. Especially important are measurable set-valued maps, e.g., set-valued random variables, random sets, probability, and statistics, but which are out of the scope of this paper. For these, see [10]. K. Menger (1942) introduced the statistical metric space as a generalization of a metric space $(M, d)$, where the distance between the elements, $p$ and $q$ of $M$, was given by a distribution function $F_{p, q} \in \Delta^{+}$(see for example [11]). The inequality:

$$
\left.F_{f x, f y}(q s) \geqslant F_{x, y}(s) \text { with } s \geqslant 0 \& q \in\right] 0,1[\text {, }
$$

is transferred for multifunctions. In this paper, we considered four extensions of the previous inequality for multifunctions. We give an overview of some F.P.T.s in probabilistic metric spaces for multivalued functions. Section 2 is devoted to the important operation of the triangular norm, and based on it, in Section 3, we introduce the notion of the probabilistic metric space (p.m.s.). We introduce in Section 5 the notion of a multivalued probabilistic $\Psi$-contraction, and by using the notion of the function of non-compactness, the first F.P.T. is given. Using the Hausdorff distance, S.B. Nadler obtained in [12] a generalization of the Banach contraction principle in metric spaces, and we extend it in Section 6 on a second F.P.T. probabilistic version. As a corollary, a Tardiff multivalued F.P.T. is obtained. Finally, a probabilistic version of Itoh's F.P.T. from [13] is given in Section 7. We present in Section 8 the fourth F.P.T. obtained by Miheţ; see [14]. In the end, we give in Section 9 some hints about some further related investigations.

\section{Triangular Norms}

The triangular norms appeared first in the framework of p.m.s. in the work of K. Menger and in the final form by Schweizer and Sklar (see for example $[11,15]$ ). This 
binary operation was used in the extension of the triangle inequality, hence the name triangular norm (t-norm). The amazing consequence was that triangular norms later appeared as important operations in many other fields, mostly modeling different types of uncertainties. In the theory of fuzzy logic, it plays the role of conjunction, and in the theory of fuzzy sets, the intersection of fuzzy sets; see [15]. Further, the triangular norm (and its extension on the extended real numbers) appeared in the theory of non-additive measures (more precisely, pseudo-additive measures) and the corresponding integrals; see [15,16]. Based on them, it was developed as an extension of the classical real mathematical analysis, under the name pseudo-analysis; see [16].

Definition 1. A function $T:[0,1]^{2} \rightarrow[0,1]$ such that the following properties hold for $x, y, z \in$ $[0,1]$ :

$\left(T_{1}\right) T(x, y)=T(y, x)$

( $\left.T_{2}\right) T(x, T(y, z))=T(T(x, y), z)$

(T3) $T(x, y) \leqslant T(x, z)$ if $y \leqslant z$

( $\left.T_{4}\right) T(x, 1)=x$

(commutativity);

(associativity);

(monotonicity);

(boundary condition),

is called a triangular norm ( $t$-norm).

Example 1. Examples of t-norms are the following:

(i) Minimum $T_{\mathbf{M}}$ where $T_{\mathbf{M}}(x, y):=\min (x, y)$;

(ii) Product $T_{\mathbf{P}}$ with $T_{\mathbf{P}}(x, y):=x \cdot y$;

(iii) Łukasiewicz t-norm $T_{\mathbf{L}}$ given by $T_{\mathbf{L}}(x, y):=\max (x+y-1,0)$.

It is easy to see that $T_{\mathbf{L}}(x, y)<T_{\mathbf{P}}(x, y)<T_{\mathbf{M}}(x, y)$ for $x, y \neq 0,1$. Moreover, a t-norm $T$ is left-continuous if for every sequence $\left(x_{n}\right)_{n \in \mathbb{N}} \subset[0,1]$ and every $y \in[0,1]$, it is:

$$
\sup _{n \in \mathbb{N}} T\left(x_{n}, y\right)=T\left(\sup _{n \in \mathbb{N}} x_{n}, y\right)
$$

The left-continuity in the point $(1,1)$ yields $\sup _{x \in[0,1[} T(x, x)=1$.

Each t-norm $T$, by associativity, can be extended in a unique way to an $n$-ary operation taking for $\left(x_{1}, \ldots, x_{n}\right) \in[0,1]^{n}, n \in \mathbb{N} . T\left(x_{1}, \ldots, x_{n}\right)$ is defined recursively by:

$$
\left\{\begin{array}{l}
\mathrm{T}_{i=1}^{0} x_{i}=1, \\
\mathrm{~T}_{i=1}^{n} x_{i}=T\left(\mathrm{~T}_{i=1}^{n-1} x_{i}, x_{n}\right)=T\left(x_{1}, \ldots, x_{n}\right) .
\end{array}\right.
$$

Specifically, $x_{T}^{(n)}$ is given recursively by the formula:

$$
\left\{\begin{array}{l}
x_{T}^{(1)}=x \\
x_{T}^{(n)}=T\left(x_{T}^{(n-1)}, x\right), \quad n \geqslant 2 \& x \in[0,1] .
\end{array}\right.
$$

Definition 2. A t-norm $T$ is of the Hadžic type (H-type) if the sequence $\left(x_{T}^{(n)}\right)_{n \in \mathbb{N}}$ is equicontinuous at $x=1$.

In the paper [14], a significant example of a t-norm $T$ such that $\left(x_{T}^{(n)}\right)_{n \in \mathbb{N}}$ is an equicontinuous family at $x=1$ was given.

We can also extend $T$ to a countable infinitary operation taking for any sequence $\left(x_{n}\right)_{n \in \mathbb{N}} \subset[0,1]$ the values:

$$
\prod_{i=1}^{\infty} x_{i}=\lim _{n \rightarrow \infty} \prod_{i=1}^{n} x_{i}
$$


A special type of t-norm $T$ is such that for a sequence $\left(x_{n}\right)_{n \in \mathbb{N}} \subset[0,1]$ with $\lim _{n \rightarrow \infty} x_{n}=1$, we have:

$$
\lim _{n \rightarrow \infty} \prod_{i=n}^{\infty} x_{i}=\lim _{n \rightarrow \infty} \prod_{i=1}^{\infty} x_{n+i}=1
$$

For a $H$-type norm $T$, (2) holds.

\section{Probabilistic Metric Spaces}

As it is well known in the theory of metric space $S$, the basic notion is a metric $d: S \rightarrow[0, \infty[$, which corresponds to every pair $(p, q)$ of elements from $S$, a non-negative number. The basic property of $d$ is the triangle inequality. Initiated by some problems with uncertainties, K. Menger introduced a generalization of the notion of the metric space. He considered the set $S$ endowed with the family of probability distribution functions $F_{p, q}$. In this way, to a pair $(p, q)$ of elements from $S$ corresponds a distribution function $F_{p, q}$. The main problem was the classical triangle inequality, which was solved by $\mathrm{t}$-norm $T$ (elaborated further by Schweizer and Sklar) in the following inequality:

$$
F_{p, r}(x+y) \geqslant T\left(F_{p, q}, F_{q, r}\right)(x, y \in \mathbb{R})
$$

for every $p, q, r \in S$.

Now, we give the precise definition of the p.m.s., introducing some notions first:

- A distance distribution function is a non-decreasing function $F:[0, \infty] \rightarrow[0,1]$ with the following properties:

(i) $F(0)=0$ and $\lim _{x \rightarrow \infty} F(x)=1$;

(ii) it is left continuous on $] 0, \infty[$.

$\mathcal{D}^{+}$denotes the family of all them;

- A triangle function $\tau$ is a commutative, associative, and non-decreasing in each place binary operation on $\mathcal{D}^{+}$that has Dirac function $H_{0}$ given by:

$$
H_{0}(v)= \begin{cases}0 & \text { if } v=0 \\ 1 & \text { if } v \in] 0, \infty]\end{cases}
$$

as the identity.

We have the following $[11,14]$ :

Definition 3. A p.m.s. is a triple $(S, \mathcal{F}, \tau)$ with $S \neq \varnothing, \mathcal{F}: S \times S \rightarrow \Delta^{+}$, which maps $(p, q) \mapsto F_{p, q}$, and $\tau$ is a triangle function, so that for every $p, q, r \in S$, the following conditions are satisfied:

(i) $F_{p, p}=H_{0}$;

(ii) $F_{p, q} \neq H_{0}$ for $p \neq q$;

(iii) $F_{p, q}=F_{q, p}$;

(iv) $F_{p, r} \geqslant \tau\left(F_{p, q}, F_{q, r}\right)$.

In particular, for $\tau=\tau_{T}$, given by:

$$
\tau_{T}\left(F^{\prime}, F^{\prime \prime}\right)(x)=\sup \left\{T\left(F^{\prime}(u), F^{\prime \prime}(v)\right) \mid u+v=x\right\}
$$

where $T$ is a $t$-norm, we have a Menger space, denoted by $(S, \mathcal{F}, T)$.

We assume in the whole paper that Range $(\mathcal{F}) \subset \mathcal{D}^{+}$.

We consider on $(S, \mathcal{F}, \tau)$ a family of neighborhoods: $\left(N_{p}(\sigma, \mu)\right)_{p \in S, \sigma>0, \mu \in] 0,1[}$ given by:

$$
N_{p}(\sigma, \mu)=\left\{q \mid q \in S, F_{p, q}(\sigma)>1-\mu\right\},
$$


and it is called a $(\sigma, \mu)$-topology. Especially for a Menger space $(S, \mathcal{F}, T)$ with $\sup _{a<1} T(a, a)=1$, the family $\left(\mathcal{N}_{p}\right)_{p \in S}$, where $\mathcal{N}_{p}=\left\{N_{p} \mid t>0\right\}$, defines on $S$ a metrizable topology.

We have in the $(\sigma, \mu)$-topology that $\left(p_{n}\right)_{n \in \mathbb{N}} \subset S$ converges to $p \in S$ if for every $\sigma>0$ and $\mu \in] 0,1\left[\right.$, there exists $n_{0}(\sigma, \mu) \in \mathbb{N}$ such that:

$$
F_{p_{n}, p}(\sigma)>1-\mu \quad \forall n \geqslant n_{0}(\sigma, \mu)
$$

(see [11]). Analogously, $\left(p_{n}\right)_{n \in \mathbb{N}} \subset S$ is a Cauchy sequence if for every $\sigma$ and $\left.\mu \in\right] 0,1[$, there exists $n_{0}(\sigma, \mu) \in \mathbb{N}$ with $F_{p_{n}, p_{m}}(\sigma)>1-\mu$, for every $n, m \geqslant n_{0}(\sigma, \mu)$. The notion of completeness for the space $S$ is the usual one.

Let $S \neq \varnothing$. The symbol $\mathcal{P}(S)$ denotes the family of all subsets of $S ; \mathcal{P}_{0}(S)$ is the family of all nonempty subsets of $S$. Related to the $(\sigma, \mu)$-topology, we recall the following families of subsets of $S$ :

- $\quad \mathcal{P}_{b}(X):=\left\{A \in \mathcal{P}_{0}(S) \mid A\right.$ is bounded $\} ;$

- $\mathcal{P}_{f}(S):=\left\{A \in \mathcal{P}_{0}(S) \mid A\right.$ is closed $\}$;

- $\mathcal{P}_{b f}(S):=\mathcal{P}_{b}(X) \cap \mathcal{P}_{f}(X)$;

- $\mathcal{P}_{c}(S):=\left\{A \in \mathcal{P}_{0}(S) \mid A\right.$ is compact $\}$.

If $S$ is a vector space, then for every $A, B \in \mathcal{P}_{0}(S)$ and every $\alpha \in \mathbb{R}$, let:

$$
A+B=\{x+y \mid x \in A, y \in B\}, \quad \alpha A=\{\alpha x \mid x \in A\} .
$$

Let $h$ be the Hausdorff metric on the metric space (with metric $d$ ) given by:

$$
h(A, B)=\max \left(\sup _{x \in A} d(x, B), \sup _{x \in B} d(x, A)\right) \quad\left(A, B \in \mathcal{P}_{0}(X)\right),
$$

and $d(x, B)=\inf _{y \in B} d(x, y)$.

\section{Fixed-Point Theorems in Probabilistic Metric Spaces}

The famous Banach contraction principle on a complete metric space $(S, d)$ says that for every $q$-contraction $f: S \rightarrow S$, i.e., if there exists a $q \in[0,1[$ such that:

$$
d(f x, f y) \leqslant q d(x, y) \text { for every } x, y \in S,
$$

there is one and only one fixed point. Sehgal and Bharucha-Reid transferred in 1972 the notion of a probabilistic $q$-contraction $(q \in] 0,1[)$ into a p.m.s.; see[14].

Definition 4. A mapping $f: S \rightarrow S$ in p.m.s. $(S, \mathcal{F})$ is a probabilistic $q$-contraction for $q \in] 0,1[$ if the following inequality holds:

$$
F_{f p_{1}, f p_{2}}(x) \geqslant F_{p_{1}, p_{2}}\left(\frac{x}{q}\right)
$$

for every $p_{1}, p_{2} \in S$ and every $x \in \mathbb{R}$.

It is easy to see that there is a generalization of the previous classical inequality since every metric space is also a Menger space $\left(S, \mathcal{F}, T_{\mathbf{M}}\right)$.

The first F.P.T. in a p.m.s. was proven by Sehgal and Bharucha-Reid; see [14].

Theorem 1. Let $\left(S, \mathcal{F}, T_{\mathbf{M}}\right)$ be a complete Menger space and $f: S \rightarrow S$ a probabilistic $q$ contraction. Then, there exists a unique fixed point $x$ of the mapping $f$ and $x=\lim _{n \rightarrow \infty} f^{n} p$ for every $p \in S$. 
The main problem in obtaining a more general F.P.T. is to find those t-norms $T$ that are strong enough to guarantee that the sequence of iterates $\left(f^{n} p\right)_{n \in \mathbb{N}}$ is a Cauchy sequence, and the other is to impose a growth condition on the distance distribution functions; see [14]. We stress this property in the following definition.

Definition 5. At-norm $T$ has the fixed-point property if and only if every probabilistic q-contraction $f: S \rightarrow S$, where $(S, \mathcal{F}, T)$ is an arbitrary complete Menger space, has a fixed point.

An important related result was obtained by V. Radu in the following theorem; see [14].

Theorem 2. Any continuous t-norm $T$ with the fixed point property is of the H-type.

Immediately, we obtain an important consequence: no Archimedean $t$-norm has the fixed-point property. Therefore, to obtain some kind of F.P.T. for Menger spaces $(S, \mathcal{F}, T)$, where $T$ is an Archimedean t-norm, one has to impose some additional conditions on the mapping $\mathcal{F}$; see [14]. We shall use these facts in treating the F.P.T. for multifunctions. Before that, we need, in the next sections, the following notions. Suppose that $A$ is a nonempty subset set of $S$. The probabilistic diameter of the set $A$ is the function $D_{A}(\cdot):[0, \infty[\rightarrow[0,1]$ given for $v \in[0, \infty[$ by:

$$
D_{A}(v):=\sup _{u<v} \inf _{p, q \in A} F_{p, q}(u) .
$$

We say that a set $A$ is probabilistic bounded if:

$$
\sup _{v \in[0, \infty[} D_{A}(v)=1
$$

For a probabilistic bounded subset $A$ of $S$, the Kuratowski function of non-compactness $\alpha_{A}(\cdot):[0, \infty[\rightarrow[0,1]$ is given by:

$$
\alpha_{A}(v):=\sup \left\{\varepsilon \mid \varepsilon>0, \exists \text { a finite family }\left\{A_{j}\right\}_{j \in J}, \text { with } A=\cup_{j \in J} A_{j}, D_{A_{j}}(v) \geqslant \varepsilon, j \in J\right\} .
$$

The Hausdorff function $\beta_{A}(\cdot):[0 . \infty[\rightarrow[0,1]$ of the set $A$ is given by:

$$
\beta_{A}(v):=\sup \left\{\varepsilon \mid \varepsilon>0, \exists \text { a finite subset } B \text { of } S \text { such that } \sup _{u<v} \inf _{x \in A} \sup _{y \in B} F_{x, y}(u) \geqslant \varepsilon\right\},
$$

where $A$ and $B$ are probabilistic bounded subsets of $S$. A subset $A$ of $S$ is probabilistic precompact if for every $\varepsilon>0$ and $\lambda \in] 0,1\left[\right.$, there exists a finite cover $\left\{A_{j}\right\}_{j \in J}$ of $A$, such that:

$$
D_{A_{j}}(\varepsilon)>1-\lambda \quad \text { for all } j \in J
$$

In the next sections, we shall represent four F.P.T.s in a p.m.s. for multifunctions. In these theorems, there are different suppositions on t-norm $T$ in $(S, \mathcal{F}, T)$, or supposed different properties of the space $(S, \mathcal{F}, T)$, or supposed different properties on considered multifunction $f$. In the first F.P.T., we extend the inequality (3) for multifunctions, introducing an additional function $\Psi$ in the inequality. In this way, we introduce a multivalued probabilistic $\Psi$-contraction, and then, using the Kuratowski function of non-compactness, we obtain by continuous t-norm of an F.P.T. In the second F.P.T., we extend Nadler's result for multifunctions on metric spaces on a p.m.s. introducing the probabilistic Nadler $q$-contraction, which for a single-valued function reduces to the classical probabilistic $q$-contraction from Definition 4. Using a special type of t-norm, it is obtained for demicompact functions' F.P.T. The third F.P.T. is devoted to closed mapping $f: S \rightarrow \mathcal{P}_{b f}$, which is densifying with respect to the function $\gamma \in\{\alpha, \beta\}$. Then, there is an F.P.T. on a probabilistic bounded subset $M$ of $S$. In the fourth F.P.T., characterization of t-norm $T$ of the $H$-type is used, and then, by it, an F.P.T. is obtained on a compact p.m.s. $(S, \mathcal{F}, T)$. 


\section{First Fixed-Point Theorem}

Using a real-valued function $\Psi:[0, \infty[\rightarrow[0, \infty[$, the single-valued $q$-contraction was extended to a multivalued probabilistic $\Psi$-contraction. Based on the notion of the function of non-compactness, we obtain an F.P.T. The following notion was introduced in [17].

Definition 6. Let $\Psi:\left[0, \infty\left[\rightarrow\left[0, \infty\left[. f: S \rightarrow \mathcal{P}_{0}(S)\right.\right.\right.\right.$ be a multivalued probabilistic ( $\left.\Psi\right)$ contraction if for every $x, y \in S$ and every $p \in f x$, there exists $q \in f y$ with:

$$
F_{p, q}(\Psi(\varepsilon)) \geqslant F_{x, y}(\varepsilon), \quad \forall \varepsilon>0 .
$$

Remark 1. Let $f: S \rightarrow S$ be a probabilistic $\Psi$-contraction for $\Psi(u)=q v(v>0), q \in] 0,1[$, then $f$ reduces on the classical probabilistic q-contraction.

Definition 7. Let $\gamma \in\{\alpha, \beta\}, g:\left[0, \infty\left[\rightarrow\left[0, \infty\left[\right.\right.\right.\right.$ and $f: M \rightarrow \mathcal{P}_{0}(S)$, where $M$ is a probabilistic bounded subset of $S$. If for every $v>0$ :

$$
\gamma_{f(A)}(g(v)) \geqslant \gamma_{A}(v)
$$

for every $A \subset M$, then $f$ is a $(\gamma, g)$-condensing mapping.

Definition 8. A mapping $f: M \rightarrow \mathcal{P}_{0}(S)(M \subset S)$ is densifying on $M$ with respect to the function $\gamma \in\{\alpha, \beta\}$ if $f(M)$ is a probabilistic bounded subset of $S$ and for every $B \subset M$ :

$$
\gamma_{f(B)}(u) \leqslant \gamma_{B}(u), \quad \forall u>0 \Rightarrow B \text { is precompact. }
$$

We denote by $\mathcal{E}$ the class of all strictly increasing mappings $\Psi:[0, \infty[\rightarrow[0, \infty[$ with the property $\lim _{n \rightarrow \infty} \Psi^{n}(x)=0$, for every $x \in[0, \infty$. The following theorem was given in [17]; see for example [11].

Theorem 3. Let $(S, \mathcal{F}, T)$ be complete with a continuous t-norm $T ; \varnothing \neq M \subset S$ is closed and probabilistic bounded and $f: M \rightarrow \mathcal{P}_{c}(M)$ a multivalued probabilistic $\Psi$-contraction. If $\Psi \in \mathcal{E}$, then there exists $x \in M$ with $x \in f x$.

\section{Second Fixed-Point Theorem}

Based on the result of Nadler on the generalization of the Banach contraction principle for multivalued mappings, we introduce the probabilistic Nadler $q$-contraction. We show how some additional properties of t-norms imply the corresponding generalizations of the F.P.T. in probabilistic metric spaces.

Let $(X, d)$ be a metric space. S.B. Nadler generalized in [12] the Banach contraction principle for $f: X \rightarrow \mathcal{P}_{b f}(X)$ by the condition:

$$
h(f x, f y) \leqslant q d(x, y),
$$

where $h$ is the Hausdorff metric for $q \in] 0,1[$.

We recall the following:

Definition 9 ([18]). Let $\varnothing \neq M \subset S$. A map $f: M \rightarrow \mathcal{P}_{0}(S)$ is a probabilistic Nadler $q$-contraction for $q \in] 0,1]$ ( $p N q$-contraction) if for every $u, v \in M, x \in f u$, and $\delta>0$, there exists $y \in f v$ such that for every $\varepsilon>0$ :

$$
F_{x, y}(\varepsilon) \geqslant F_{u, v}\left(\frac{\varepsilon-\delta}{q}\right) .
$$




\section{Remark 2.}

(i) For a single-valued function, the introduced probabilistic Nadler $q$-contraction from Definition 9 reduces to the notion of a probabilistic $q$-contraction given by Sehgal and Bharucha-Reid (see [14]), and (4) reduces to:

$$
F_{f u, f v}(\varepsilon) \geqslant F_{u, v}(\varepsilon) \quad \forall u, v \in M \quad \& \quad \forall \varepsilon>0 ;
$$

(ii) If we assume $q=1$ in (4), $f$ is a multivalued probabilistic non-expansive mapping of the Nadler type;

(iii) If $(S, d)$ is a metric space and $f: M \rightarrow \mathcal{P}_{b f}(S)$ is a $q$-contraction in the sense of Nadler [12], then $f$ is also a probabilistic Nadler $q$-contraction in the corresponding Menger space $\left(S, \mathcal{F}, T_{\mathbf{M}}\right)$.

The next example is related to random operators.

Example 2. Let $X$ be a separable metric space, $(\Omega, \mathcal{A}, P)$ a probability space, and $\left(S, \mathcal{F}, T_{\mathbf{L}}\right)$ the Menger space of all classes of equivalence of measurable functions from $\Omega$ into $X$.

Let $f: \Omega \times X \rightarrow \mathcal{P}_{b f}(M)$ be a random operator, i.e., for every $x \in X$, the function $f(\cdot, x): \Omega \rightarrow \mathcal{P}_{b f}(X)$ is measurable, namely for every open $O \subset X:$

$$
f^{-1}(O)=\{\omega \in \Omega \mid f(\omega) \cap O \neq \varnothing\} \in \mathcal{A} .
$$

Suppose that, for every $x, y \in X$ and every $\omega \in \Omega$ :

$$
h(f(\omega, x), f(\omega, y)) \leqslant q d(x, y)(q \in] 0,1[) .
$$

For every measurable function $R: \Omega \rightarrow X$, the function $\omega \mapsto f(\omega, R(\omega))$ is measurable, and therefore, there exists a Castaing representation $\left(u_{n}\right)_{n \in \mathbb{N}}$ of measurable selectors such that:

$$
f(\omega, R(\omega))=\overline{\left\{u_{n}(\omega) \mid n \in \mathbb{N}\right\}} .
$$

Let $\bar{f} R:=\{U \mid U \in S, U(\omega) \in f(\omega, R(\omega))$ for every $\omega \in \Omega\}$. It is easy to see that the function $\bar{f}$ is a probabilistic Nadler $q$-contraction in the Menger space $\left(S, \mathcal{F}, T_{\mathbf{L}}\right)$; see [11,14].

Definition 10. Suppose that $(S, \mathcal{F})$ is a p.m.s., $\varnothing \neq M \subset S$ and $f: M \rightarrow \mathcal{P}_{0}(S)$. We say that a function $f$ is weakly demicompact if every sequence $\left(x_{n}\right)_{n \in \mathbb{N}} \subset M$ such that:

$$
\begin{cases}x_{n+1} \in f x_{n}, & \text { for every } n \in \mathbb{N} \text { and } \\ \lim _{n \rightarrow \infty} F_{x_{n+1}, x_{n}}(\varepsilon)=1, & \text { for every } \varepsilon>0\end{cases}
$$

admits a convergent subsequence $\left(x_{n_{k}}\right)_{k \in \mathbb{N}}$.

We have by [14] (Theorem 4.13) the following:

Theorem 4. Suppose that $(S, \mathcal{F}, T)$ is complete, $T$ a t-norm with $\sup T(a, a)=1$, and $\varnothing \neq$ $M \subset S$. If $f: M \rightarrow \mathcal{P}_{f}(M)$ is a probabilistic Nadler $q$-contraction, $\left.q \in \underset{q}{a} \in\right] 0,1[$, with the following properties:

(i) $f$ is weakly demicompact;

(ii) There are $x_{0} \in M, x_{1} \in f x_{0}$, and $\left.v \in\right] q, 1[$ such that:

$$
\lim _{n \rightarrow \infty} \prod_{i=n}^{\infty} F_{x_{0}, x_{1}}\left(\frac{1}{v^{i}}\right)=1
$$

then there exists $x \in M$ with $x \in f x$. 
Corollary 1. Suppose that $(S, \mathcal{F}, T)$ is complete, $T$ a H-type norm and $\varnothing \neq M \subset S$. If $f: M \rightarrow$ $\mathcal{P}_{f}(M)$ is a pNq-contraction, $\left.q \in\right] 0,1[$, then there exists $x \in M$ with $x \in f x$.

Corollary 2. Suppose that $(S, \mathcal{F}, T)$ be complete, $T$ a t-norm such that $T \geqslant T_{L}$, and $\varnothing \neq M \subset S$. If $f: M \rightarrow \mathcal{P}_{f}(M)$ is a $p N q$-contraction, $\left.q \in\right] 0,1\left[\right.$, and if there exist $x_{0} \in M$ and $x_{1} \in f x_{0}$ with:

$$
\int_{1}^{\infty} \ln u d F_{x_{1}, x_{0}}(u)<\infty,
$$

then there exists $x \in M$ with $x \in f x$.

\section{Third Fixed-Point Theorem}

We extend the classical result Itoh obtained for multivalued mappings on complete metric spaces, on a probabilistic version, with a general continuous t-norm.

Definition 11. We call a function $f: X \rightarrow \mathcal{P}_{b f}(X)$ closed on $S$ if for every sequence $\left(x_{n}\right)_{n \in \mathbb{N}}$ from $S$ and every sequence $\left(y_{n}\right)_{n \in \mathbb{N}}$ such that $y_{n} \in f x_{n}$, for every $n \in \mathbb{N}$, we have that $x_{n} \rightarrow x, y_{n} \rightarrow y$ implies $y \in f x$.

The paper [19] is devoted to an F.P.T. for multivalued mappings on complete metric space $(X, d)$.

Theorem 5 (Itoh [19]). If $f: X \rightarrow \mathcal{P}_{b f}(X)$ is an upper semi-continuous mapping, $\varnothing \neq B \subset X a$ bounded set such that $f(B)$ is also bounded, and the following conditions hold:

(i) $\inf _{x \in B} d(x, f x)=0$;

(ii) For every $A \subset B$, we have that $\alpha(A)>0 \Rightarrow \alpha(f(A))<\alpha(A)$;

then there exists $x \in \bar{B}$ such that $x \in f x$.

We proved a probabilistic metric version of Theorem 5 for complete Menger space $(S, \mathcal{F}, T)$ with a continuous t-norm $T$ in [14].

Theorem 6 ([14]). If $f: S \rightarrow \mathcal{P}_{b f}(S)$ is a closed mapping and there exists a non-empty probabilistic bounded subset $M$ of $S$ such that $f(M)$ is also probabilistic bounded and the following conditions hold:

(i) There exist two sequences $\left(x_{n}\right)_{n \in \mathbb{N}} \subset M$ and $\left(y_{n}\right)_{n \in \mathbb{N}}$ such that for every $n \in \mathbb{N}, y_{n} \in f x_{n}$ and

$$
\lim _{n \rightarrow \infty} F_{x_{n}, y_{n}}(\varepsilon)=1 \quad \text { for every } \varepsilon>0 ;
$$

(ii) The mapping $f$ is densifying on $M$ with respect to the function $\gamma$, where $\gamma \in\{\alpha, \beta\}$;

then there exists at least one element $x \in \bar{M}$ such that $x \in f x$.

\section{Fourth Fixed-Point Theorem}

Based on a characterization of the t-norm of the Hadžić type, a p.m.s. version of the F.P.T. on the Menger space with a t-norm of the $H$-type was obtained. Let $(S, \mathcal{F}, T)$ be a Menger space.

Definition 12. The probabilistic distance between $A$ and $B$ from $\mathcal{P}_{f b}(S)$, for $\widetilde{F}_{A, B}: \mathbb{R} \rightarrow[0,1]$, is given by:

$$
\widetilde{F}_{A, B}(u)=\sup _{s<u} T\left(\inf _{p \in A} \sup _{q \in B} F_{p, q}(s), \inf _{q \in B} \sup _{p \in A} F_{p, q}(s)\right), \quad u \in \mathbb{R} .
$$


We have the following characterization: $T$ is of $H$-type if and only if there exists a non-decreasing sequence $\left.\left(b_{n}\right)_{n \in \mathbb{N}} \subset\right] 0,1\left[\right.$ with $\lim _{n \rightarrow \infty} b_{n}=1$ and for every $n \in \mathbb{N}$, we have that:

$$
b_{n}<x \leqslant 1, b_{n}<y \leqslant 1 \Rightarrow b_{n}<T(x, y) \text {. }
$$

Theorem 7 (D. Miheţ in [14,20]). Suppose that $(S, \mathcal{F}, T)$ is compact, $T$ is of the H-type, and $f: S \rightarrow \mathcal{P}_{b f}(S)$. If for every $n \in \mathbb{N}$ there exists a $\left.k_{n} \in\right] 0,1[$ such that for every $p, q \in S$ and every: $s>0$,

$$
F_{p, q}(s)>b_{n} \Rightarrow \widetilde{F}_{f p, f q}\left(k_{n} s\right)>b_{n},
$$

then there is $x \in S$ with $x \in f x$.

\section{Further Investigations}

There are also some other types of fixed-point results for multivalued maps on probabilistic spaces. One such type was obtained by T. Hicks in [21,22]. We generalized the notion of the $C$-contraction for the multivalued case in [14], and we proved two F.P.T.s for multivalued mappings.

In the paper [23], some general fixed- and common fixed-point results for multivalued $\mathcal{H}^{+}$-type contraction mappings in symmetric spaces were obtained, and as an application, results in probabilistic spaces were obtained.

The paper [24] is devoted to a complete Menger space with an $H$-type norm. As a consequence, some previously proven F.P.T.s were obtained, as well as a convergence result of successive approximations for suitable nonlinear operators. As a consequence, a generalization of Kelisky-Rivlin's result was obtained [25] (each Bernstein operator is a weak operator on the space $C([0,1]))$ for a class of modified $q$-Bernstein operators on the space, which for $q>0$ is based on the $q$-factorial $[n]_{q} !:=[1]_{q}[2]_{q} \cdots[n]_{q}(n=1,2, \ldots)$ and $[0]_{q} !:=1$, where $[n]_{q}:=1+q+q^{2}+\cdots+q^{n-1}$.

In the paper [26], a new definition of a class of contractions in the multivalued case was given, and some F.P.T.s for multivalued $(\psi, \psi, \varepsilon, \lambda)$ - contraction mappings in the probabilistic metric space were proven.

\section{Conclusions}

We gave an overview of four important extensions of the F.P.T. for multivalued functions in probabilistic metric spaces. We mentioned some further investigations into the considered topic.

There are many important open problems related to the p.m.s. in [11] and to the theory of triangular norms [27]. Some of them have already been solved, but some of them are still open.

Since there are many open problems, we mention here only two of them. One is related to the F.P.T. in the topological vector space; see [14] (Chapter 6). Namely, it is known that a random normed space $(S, \mathcal{F}, T)$ for a continuous t-norm $T$ is a topological vector space (t.p.s) that is not always a locally convex space. If the continuous t-norm is of the $H$-type, then it is a locally convex space. Therefore, the general open problem of the F.P.T. is related to Schauder's conjecture: every compact convex subset of a linear metric space has the fixed-point property. Unfortunately, this problem is still open. Tychonov generalized Brower's F.P.T. (every continuous map on a non-empty convex and compact subset of $\mathbb{R}^{n}$ has at least one fixed point) to the locally convex space. It is still an open problem whether this true also for the general vector space. The notion of admissibility is very important in such investigations. Let $X$ be a t.p.s. and $\mathcal{U}$ be the fundamental system of neighborhoods of $0 \in X$. We say that $X$ is admissible if for every compact subset $K$ of $X$ and every $U \in \mathcal{U}$, there exists a continuous mapping $\varphi: K \rightarrow X$ such that the dimension of the linear hull of $\varphi(K)$ is finite and for every $x \in K$, we have $x-\varphi(x) \in U$. For example, the space $L^{p}(0,1)$ for $0<p<1$ is admissible. It is still an open problem whether there exists a convex non-admissible subset of a t.v.s. This investigation admissibility gives that $\left(S(\Omega, \mathcal{A}, P), \mathcal{F}, T_{\mathbf{L}}\right)$ is an admissible t.v.s., which is a random normed space. Then, the 
approach with admissibility gives the F.P.T. for this space. However, we shall not go further into the explanations; see [14] (Chapter 6).

A second important topic is related to the dominance relation. We say that t-norm $T^{*}$ dominates a t-norm $T, T^{*} \gg T$, if for each $a, b, c, d \in[0,1]$,

$$
T^{*}(T(a, b), T(c, d)) \geqslant T\left(T^{*}(a, c), T^{*}(b, d)\right) .
$$

It is obvious that for any t-norm $T$, it holds that $T_{\mathbf{M}} \gg T$. The construction of the Cartesian product of the p.m.s. is based on the domination relation; see [11,15]. Since it is obvious that the domination relation is reflexive and symmetric, a question arises about the transitivity, i.e.: Is the domination relation always a partial order, on the set of all t-norms? If not, for what subsets is this the case? H. Sherwood gave a counterexample for the general question; see [28].

Funding: Supported by the Science Fund of the Republic of Serbia, Grant No. 6524105, ATLAS-AI.

Conflicts of Interest: The author declares no conflict of interest.

\section{References}

1. Agarwal, P.; Jleli, M.; Samet, B. Fixed Point Theory in Metric Spaces: Recent Advances and Applications; Springer: Berlin, Germany, 2018.

2. Pathak, H.K. An Introduction to Nonlinear Analysis and Fixed Point Theory; Springer: Berlin, Germany, 2018.

3. Kirk, W.A.; Sims, B. Handbook of Metric Fixed Point Theory; Springer: Berlin, Germany, 2001.

4. Dugundji, J.; Granas, A. Fixed Point Theory; Springer: Berlin, Germany, 2003.

5. Brown, R.F. (Ed.) Fixed Point Theory and Its Applications; American Mathematical Society: Providence, RI, USA, 1988.

6. Aubin, J.P.; Frankowska, H. Set-Valued Analysis; Birkhäuser: Boston, MA, USA, 1990.

7. Hu, S.; Papageorgiou, N.S. Handbook of Multivalued Analysis; Kluwer Academic Publishers: Dordrecht, The Netherlands, 1997; Volume I.

8. Hildebrand, W. Core and Equilibria of a Large Economy; Princeton University Press: Princeton, NJ, USA, 1974.

9. Hu, S.; Papageorgiou, N.S. Handbook of Multivalued Analysis, Vol. II (Applications); Kluwer Academic Publishers: Dordrecht, The Netherlands, 1990.

10. Gavriluţ, A.; Pap, E. Regular Non-Additive Multimeasures. Fundaments and Applications; Studies in Systems, Decision and Control; Springer: Cham, Switzerland, 2022.

11. Schweizer, B.; Sklar, A. Probabilistic Metric Spaces; Elsevier: Dordrecht, The Netherlands; New York, NY, USA, 1983.

12. Nadler, S.B. Multivalued contraction mappings. Pac. J. Math. 1969, 30, 475-478. [CrossRef]

13. Itoh, S. A random fixed-point theorem for a multivalued contraction mapping. Pac. J. Math. 1977, 68, 85-90. [CrossRef]

14. Hadžić, O.; Pap, E. Fixed Point Theory in Probabilistic Metric Spaces; Kluwer: Dordrecht, The Netherlands; Boston, MA, USA; London, UK, 2001.

15. Klement, E.P.; Mesiar, R.; Pap, E. Triangular Norms; Kluwer: Dordrecht, The Netherlands; Boston, MA, USA; London, UK, 2000.

16. Pap, E. Null-Additive Set Functions; Kluwer Academic Publishers: Dordrecht, The Netherlands, 1995.

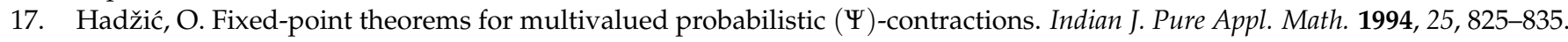

18. Hadžić, O. Fixed-point theorems for multivalued mappings in probabilistic metric spaces. Mat. Vesnik 1979, 3, 125-133. [CrossRef]

19. Itoh, S. Some fixed-point theorems in metric spaces. Fund. Math. 1979, 102, 109-117. [CrossRef]

20. Pai, D.V.; Veeramani, P. Fixed-point theorems for multimappings. Yokohama Math. J. 1980, 28, 7-14.

21. Hicks, T.L. Fixed-point theory in probabilistic metric spaces. Univ. Novom Sadu Rad. Prirod.-Mat. Fak. Ser. Mat. 1983, 13, 63-72.

22. Hicks, T.L. Fixed-point theorems for multivalued mappings. II. Indian J. Pure Appl. Math. 1998, $29,133-137$.

23. Patle, P.; Patel, D.; Aydi, H.; Radenović, S. 3ON H+Type Multivalued Contractions and Applications in Symmetric and Probabilistic Spaces. Mathematics 2019, 7, 144. [CrossRef]

24. Argoubi, H.; Jleli, M.; Samet, B. The study of fixed points for multivalued mappings in a Menger probabilistic metric space endowed with a graph. Fixed Point Theory Appl. 2015, 2015, 113. [CrossRef]

25. Kelisky, R.P.; Rivlin, T.J. Iterates of Bernstein polynomials. Pac. J. Math. 1967, 21, 511-520. [CrossRef]

26. Beitollahi, A.; Azhdari, P. Multi-valued $(\psi, \phi, \varepsilon, \lambda)$-contraction in probabilistic metric space. Fixed Point Theory Appl. 2012. Available online: http:/ /www.fixedpointtheoryandapplications.com/content/2012/1/10 (accessed on 8 February 2012).

27. Klement, E.P.; Mesiar, R.; Pap, E. Problems on triangular norms and related operators. Fuzzy Sets Syst. 2004, 145, 471-479. [CrossRef]

28. Alsina, C.; Frank, J.; Schweizer, B. Associative Functions on Intervals: Triangular Norms and Copulas; World Scientific Publishing Co.: Singapore, 2006. 